\title{
PLACE DE L'ADJECTIF BEAU DANS LA CHANSON ET LE RAP FRANCAIS
}

\author{
Dr. Miroslav Stasilo \\ Académie militaire de Lituanie du général Jonas Zemaitis \\ Universite de Vilnius \\ francaisprancuzas@yahoo.fr
}

\begin{abstract}
Sommaire. Cette étude traite les occurrences de l'adjectif qualificatif " beau », très répondu et utilisé dans la langue française. En se basant sur le corpus numérique, qui inclue une base de données d'environ 122000 mots avec 41000 vocables pour la chanson et 81000 mots pour le rap français, l'article présente la variété des usages et des différences de cet adjectif qualificatif concernant ses fonctions syntaxiques et les valeurs sémantiques qu'ils acquièrent dans le discours musical français. Pour l'analyse des occurrences, le logiciel « Notepad ++ " a été employé pour un traitement lexicométrique des 240 singles les plus populaires et vendus en France entre 1989 et 2019 (4 chansons pour chaque année). Les résultats de cette recherche ont démontré que l'adjectif «beau » était plus variable que d'autres adjectifs non seulement comme un outil propice à exprimer une qualité particulière de l'objet mais aussi comme un constituant inséparable de son syntagme nominal. Cette étude a également présenté une place variable de l'adjectif, placé généralement avant le nom, dans le genre du rap français.
\end{abstract}

Mots-clés: adjectif qualificatif, chanson, rap, analyse quantitative, champs sémantique, analyse syntaxique

\section{Introduction}

Le but de cet article est d'analyser et de classifier les occurrences de l'adjectif « beau » qu'on retrouve dans le discours musical poétique français actuel, notamment la classification des faits linguistiques de la production ainsi que de l'apparition de l'adjectif concerné dans les paroles des chansons les plus populaires en France entre 1989 et 2019. Ce travail vise également une étude concernant l'emploi de cet adjectif dans deux genres musicaux - la chanson française et le rap français. Cela nous permettra de réaliser cinq objectifs principaux : 1) recueillir toutes les occurrences des locutions adjectives de beau ; 2) classer les formes recueillies selon les critères formels ; 3) analyser l'emploi des structures distinguées et les valeurs qu'elles acquièrent ; 4) comparer le contexte d'utilisation de cet adjectif ; 5) évaluer les tendances de l'emploi dans deux genres musicaux. 
Pour atteindre ces objectifs, nous nous sommes appuyés sur des méthodes descriptive, quantitative, qualitative et comparative. Nous avons eu également recours à l'analyse lexicale quantitative grâce au logiciel Notepad ++ et grammaticale en nous basant sur des œuvres d' Elizaveta Référovskaïa et Alla Vassilieva, d'Ekaterina Nikolsaïa et Natalija Goldenberg, de Michel Pougeoise, de Gérard Ganette et de Maurice Grevisse. Or, la base théorique de nos recherches se repose sur les traditions françaises de l'analyse du discours, notamment celles de Patrick Charaudeau et Dominique Maingueneau.

\section{Adjectif qualificatif « beau »}

Étymologiquement, l'adjectif beau vient du latin classique bellus [« jolie »] (cf. : https://larousse.fr/dictionnaires/francais), qui est devenu très fréquent en bas latin en remplaçant le classique pulcher [« beau, belle »] (ibid.). Vers 980, l'adjectif beau était un vocable employé surtout par des parents chaleureux vise-à-vis de leurs enfants. Par contre, on disait « fillastre » pour un beau-fils, « marastre »- pour une belle-mère, «parastre » - pour un beau-père. Une telle appellation avait une connotation négative à l'époque. Ainsi, le suffixe -âtre a pris plus tard une signification péjorative et les mots ci-contre ont été remplacés au XII ${ }^{m e}$ siècle par des allocutions plus neutres ayant l'adjectif beau-/belle- (ibid.). Le mot beau signifie « agréable à voir ou à entendre » (ibid.). L'adjectif beau a une connotation positive qui exprime une qualité esthétique, par ex., " Mon chat est beau ». Il a plusieurs synonymes et les plus proches sont : bon, agréable, joli, grand, charmant, parfait, merveilleux, aimable, supérieure, étonnant, distingué, remarquable, gracieux, excellent formidable, etc. (cf. : http://www.crisco.unicaen.fr/des/).

L'adjectif « beau » est un adjectif qualificatif. Selon Ekaterina Nikolskaïa et Natalija Goldenberg, les adjectifs qualificatifs expriment une qualité à un degré directement par le sens de leur radical (Nikolskaïa, Goldenberg, 1974, 62). Le « beau » a des degrés d'intensité différents : égalité, supériorité et intériorité (Grevisse, 2003). La position de supériorité « $<\ldots>$ se forme au moyen de l'adverbe plus précédant l'adjectif» (ibid., 98). Le cas de l'infériorité «<...> se forme au moyen de l'adverbe moins précédant l'adjectif » (ibid.). L'intensité élevée peut être exprimée grâce à la présence des adverbes « très, tout, fort, bien, tout à fait »ou en ajoutant des préfixes ou suffixes « archi-, extra-, hyper-, -issime »; l'intensité moyenne est formée par la présence des adverbes « assez, moyennement »; les adverbes « peu, à peine » servent à présenter une intensité faible.

\section{Discours musical}

La qualité esthétique de l'adjectif « beau » est liée à l'objectif et l'action du discours musical qui se focalise sur l'éveil d'une émotion auprès de son locuteur : «<...> toute musique ne commence à avoir un effet magique qu'à partir du moment 
où nous entendons parler en elle le langage de notre propre passé : et en ce sens, pour le profane, toute musique ancienne semble devenir toujours meilleure, et toute musique récente n'avoir que peu de valeur : car elle n'éveille pas encore la 'sentimentalité', qui <...> est le principal élément de bonheur dans la musique, pour tout homme qui ne prend pas plaisir à cet art purement en artiste. » (Nietzsche, 1990, 892).

Selon le « Dictionnaire d'analyse du discours », tous les discours ont des caractéristiques de base : "Le discours suppose une organisation transphrastique $<\ldots>$ est orienté $<\ldots>$ une forme d'action $<\ldots>$ interactif $<\ldots>$ est contextualisé $<\ldots>$ est régie des normes $<\ldots>$ est pris dans un interdiscours » (Charaudeau, Maingueneau, 2002, 185 ). Ces caractéristiques sont propres aussi au discours musical : on y retrouve la mobilisation des structures d'un certain ordre, par ex., un rapport syntaxique entre les mots, le rythme et la mélodie ; ce discours vise un locuteur susceptible d'écouter ou d'aimer la musique; le traitement de ce discours n'est pas envisageable sans attention au contexte et aux liens avec d'autres type de discours, par ex., le discours poétique car dans chaque culture, la parole chantée prend la première place en tant que le plus ancien mode d'expression poétique.

Selon Gérard Ganette, « La musique et la littérature ne sont ni parallèles ni symétriques : ce sont deux pratiques simplement différentes (rien de plus difficile à penser qu'une simple différence), qui ne se rencontrent heureusement qu'en vertu de leur différence. » (Ganette, 1987, 120) Selon Anne Claire Gignoux, ce qui est généralement critiqué pour l'analogie entre la musique et la littérature, "c'est d'être gratuite, métaphorique et illégitime » (Ganette cité par Gignoux 2016, 10). Ce deux formes artistiques font des signes qui se combinent : «<... en un énoncé au déroulement temporel, à l'image de ceux du langage verbal » (ibid.). Si l'on maîtrise un minimum le code, la signification de ces deux formes sémiotiques ne peut être totalement comprise (ibid.). Le même principe est actuel pour le langage verbal : " un locuteur ignorant le chinois n'entendra rien d'un texte écrit dans cette langue » ; « un élève à la syntaxe et au lexique limités ne comprendra rien à un poème de Mallarmé » (ibid.). L'analogie entre ces deux langages, verbal et musical, nous permet de nous appuyer sur une approche lexicale et grammaticale en légitimant ainsi notre analyse quantitative et qualitative du corpus.

\section{Analyse quantitative de l'adjectif « beau »}

Nous avons compté 87 occurrences de l'adjectif beau dans les paroles de 40 chansons françaises sur 120 , ce qui fait $0,0021 \%$ de tous les textes, et 82 occurrences dans 35 paroles de rappeurs français sur 120 textes analysés, soit 0,0020\% des cas. En totalité, nous avons compté 169 occurrences de l'adjectif beau, c'està-dire, 75 textes sur 240 contiennent ce vocable, ce qui fait $0,0014 \%$ de toutes les paroles analysées : 


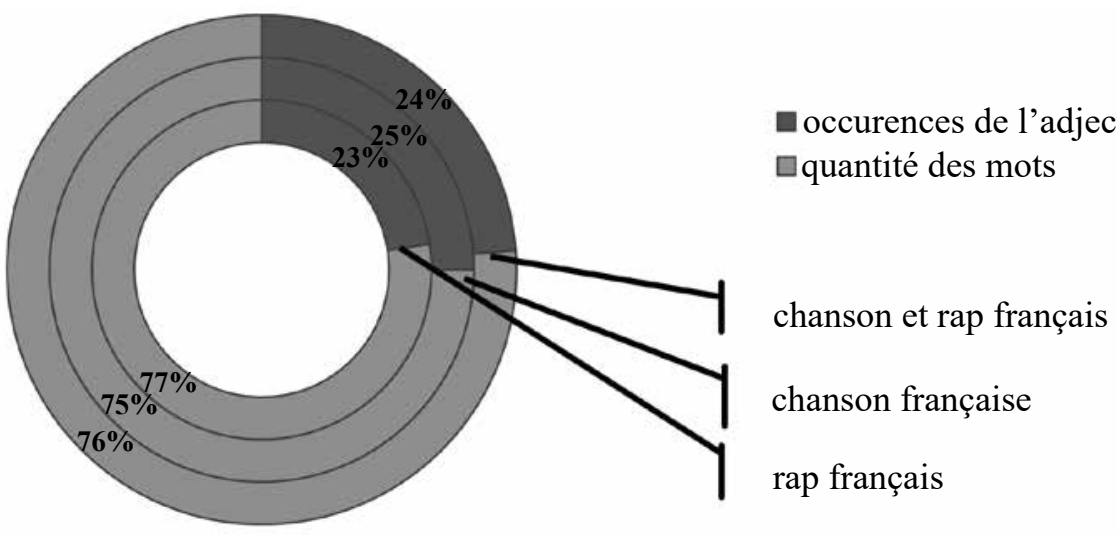

Figure 1. Répartition générale de beau dans la chanson et le rap français (1989-2019)

Comme on le voit dans le diagramme en beignet (cf. Figure 1), la distribution de la structure adjectivale beau est presqu'égale dans les deux genres analysés : la chanson en possède $25 \%$ et le rap français - $23 \%$ sur l'ensemble des cas. Le vocable beau représente par conséquent $24 \%$ de tous les vocables, ce qui démontre son emploi massif dans la chanson et le rap français.

Etant donné le pourcentage élevé de l'utilisation de beau dans les deux genres du discours musical, nous nous sommes intéressés également à sa fonction syntaxique: épithète, attribut, déterminant prédicatif ou autre :

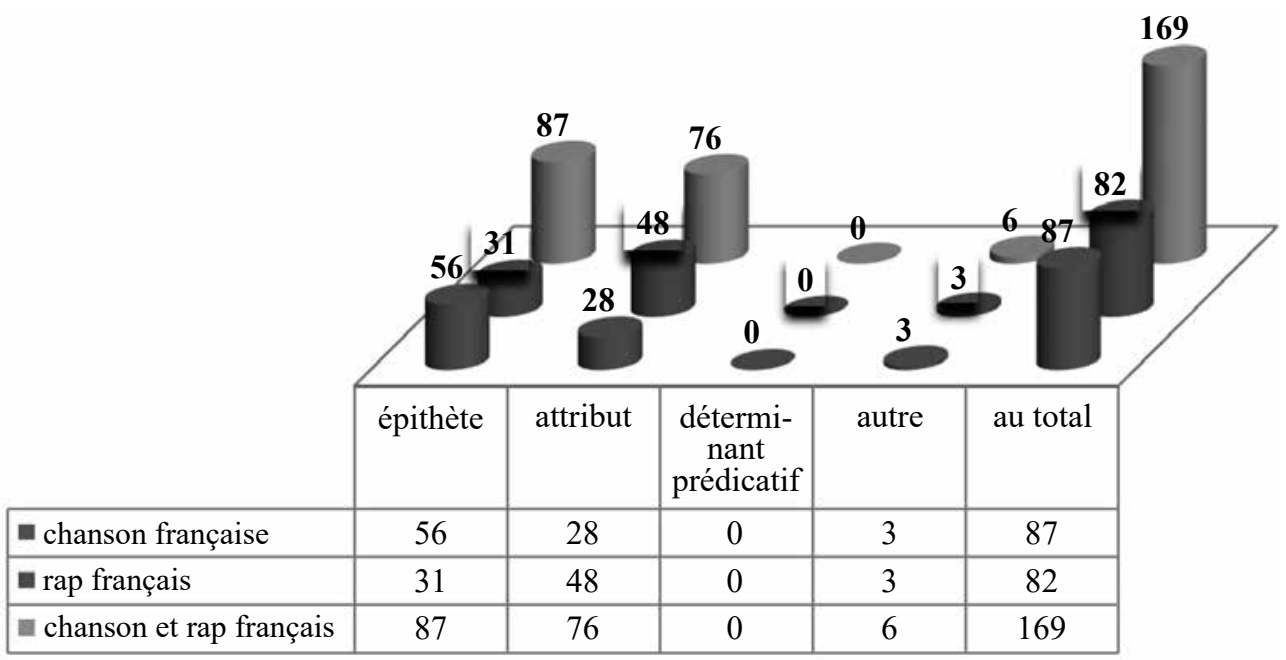

Figure 2. Répartition des fonctions de beau dans la chanson et le rap français (1989-2019)

On voit que la domination « incontestable » n'existe pas concernant la répartition quantitative de beau comme une épithète et un attribut : 87 occurrences des cas où cet adjectif joue le rôle d'épithète et 76 occurrences des situations où on le 
retrouve en tant qu'attribut du sujet ou du complément d'objet sur l'ensemble du corpus analysé. La Figure 2 nous montre aussi le «sous-emploi » de cet adjectif dans une autre fonction linguistique avec 6 occurrences seulement (cf. Figure 2) et son absence en tant que déterminant prédicatif (ibid.).

Comme l'épithète et l'attribut ont la tendance de se rattacher au nom, nous avons voulu voir la distribution quantitative des champs sémantiques de l'adjectif beau dans les deux genres. Nous présentons d'abord les champs les plus représentatifs de la chanson et ensuite du rap français, énumérés selon un ordre décroissant et appelés facultativement :

Tableau 1. Champs sémantiques dans la chanson française

\begin{tabular}{|c|l|}
\hline $\begin{array}{c}\text { Champ } \\
\text { sémantique }\end{array}$ & \multicolumn{1}{c|}{ Exemples } \\
\hline Noms propres & beau Noël (7 cas), belle Andalouse (4), belle Espagnole (4), belle Séverine \\
\hline Corps & beaux yeux (5), beau sourire (2), beau visage, belle vue \\
\hline Air & beau jour (8), beau temps (2), belle(s) histoire(s) (2), belle humeur \\
\hline Univers artistique & belles notes (2), bel objet \\
\hline Autres & belles croupes, beau parleur \\
\hline
\end{tabular}

Le Tableau montre que l'adjectif beau est majoritairement employé avec les compléments appartenant au champ des noms propres ou en collocation avec les substantifs comptables tels que « yeux, jour, notes, croupes » (cf. Tableau 1). D'un côté, on assiste donc à l'esthétisation d'une sous-catégorie de noms distincte de l'espèce, par ex., Noël en tant que fête religieuse ou Séverine comme un prénom féminin. De l'autre côté, on remarque la volonté de la mise en valeur des éléments simples, voire quotidiens, comme un sourire, des yeux ou une histoire.

En appliquant les mêmes principes quantitatifs d'analyse, nous avons étudié les paroles du rap français, dont le passé n'était pas aussi riche que celui de la chanson, mais qui a cependant beaucoup de traits en commun, notamment la distribution selon des champs sémantiques similaires, par ex., « univers artistiques », « air » et « corps » (cf. ci-dessous) :

Tableau 2. Champs sémantiques dans le rap français

\begin{tabular}{|c|l|}
\hline $\begin{array}{c}\text { Champ } \\
\text { sémantique }\end{array}$ & \multicolumn{1}{c|}{ Exemples } \\
\hline Gens & $\begin{array}{l}\text { belles puttes (3), belle femme, belles gazelles, belles blondes, belles doudous, } \\
\text { beau gosse, beaux gens }\end{array}$ \\
\hline Univers artistique & belle mélodie (6), belle plume \\
\hline Autres & beaux pulls (4), belles voitures, belle parc \\
\hline Air & beau matin (2), belle vie (2) \\
\hline Corps & belles jambes, beaux yeux, belle voix \\
\hline
\end{tabular}


Ce tableau présente de même un autre champs sémantique, celui de gens, où l'attention et la mise en relief du substantif « femme » est évidente (cf. Tableau 2). On remarque également que dans le rap français, l'adjectif beau est majoritairement employé avec les vocables qui n'entrent pas que dans le registre populaire mais aussi un langage vulgaire ou familier, par ex., putes, blondes, plume, gosse, etc. Tout cela s'explique par un contact très proche de ce genre, né au début des années 1970 dans les ghettos noirs de grandes villes américaines, avec la vie quotidienne (Marquet, 2013).

\section{Analyse syntaxique de l'adjectif « beau »}

Comme on l'a vu ci-dessus, l'emploi massif de l'adjectif beau est lié à sa fonction d'épithète ou d'attribut dans des champs sémantiques assez variés : noms propres, univers artistique, parties du corps, moments de la vie quotidienne (météo, nature, gens). Nous nous sommes également intéressés aux formes syntaxiques de cet adjectif dans les deux genres. Les exemples ci-dessous illustrent des collocations typiques dans la chanson française :

(1) Tu es mon plus beau Noël. (Johnny Hallyday, Mon Plus Beau Noël);

(2) La belle Séverine me regardera-t-elle? (Colonel Reyel, Toutes Les Nuits) ;

(3) Les beaux yeux de la Mama. (Kendji Girac, Les Yeux de la Mama);

(4) Le bleu du ciel un beau sourire du bout des lèvres. (Ilona Mitrecey, Un Monde Parfait) ;

(5) Un beau jour, ou peut-être une nuit? (Patrick Bruel, L'Aigle noir) ;

(6) De belles histoires, tu parles que des conneries! (Patrick Bruel, Qui a le droit)

(7) Alors, je me chante mes plus belles notes. (Christophe Maé, Il est où le bonheur) ;

(8) Suis-je un bel objet trop fragile. (Elsa Lunghini, Bouscule-moi);

(9) Tu as les plus belles croupes. (Moos, Au nom de la rose) ;

(10) On dit que chez toi les hommes sont tous beaux parleurs, et alors? (Pascal Obispo, Fan).

Les extraits ci-dessus montrent que l'adjectif beau précède plus souvent un objet direct qu'un sujet. Le complément d'objet direct est obligatoire dans la phrase s'il sert à représenter une qualité ou une manière d'être. Si l'on supprime l'adjectif beau, la phrase reste inachevée. Par ex., « Tu es mon ... Noël » (cf. nº 1), c.-à-d., la personne dont on parle est comme une fête parmi d'autres fêtes ; ou « On dit que 
chez toi les hommes sont tous ... des parleurs, et alors? » (cf. $\left.n^{\circ} 1\right)$, c.-à-d., les gens sont de simples parleurs. Les deux exemples démontre que l'adjectif beau forme la même unité lexicale avec son substantif.

On verra si l'adjectif beau joue le même rôle dans le rap français. Les exemples ci-dessous représentent les occurrences typiques dans les textes traités :

(11) Les belles Weston, les Aston Martin, les beaux pulls et les belles putes. (Oxmo Puccino et Lino, La Loi du point final);

(12) La monnaie est une belle femme qui n'épouse pas les pauvres. (IAM, Nés sous la même étoile);

(13) Musique t'es ma «Milady», sans belle mélodie. (Oxmo Puccino et Lino, J'ai mal au mic);

(14) C'est vrai que t'as une belle plume, ce que t'écris ça vient du coeur. (La Fouine, La Fouine vs Laouni);

(15) Qui voit les gens passer, les belles voitures passé. (Rohff, Génération sacrifiée) ;

(16) Un beau matin j'ai dit " bon, j'arrête ». (La Cliqua, Un dernier jour sur terre) ;

(17) Ses jambes, fines, belles, bonnes, longues, rondes, dodues, jolies. (Daddy Lord C, Freaky Flow);

(18) Devant leurs beaux yeux un morceau d'Oxmo ne vaut pas Mozart. (Youssoupha, Éternel recommencement);

(19) Beau gosse comme ton fils, malgré le train de vie, tu le ramenais faire du poney. (Rohff, Regretté) ;

(20) De transmission orale auprès de gens beaux et fiables. (Kery James, 28 décembre 1977).

Comme on le voit, la position dominante ici est aussi une antéposition. C'est pourquoi la plus grande majorité des occurrences porte la même valeur et signification dans les deux genres analysés - un lien moins fort avec un verbe qu'avec un substantif dont l'intensité est fortifiée et le signifié se trouve dans l'adjectif en formant ainsi le même syntagme nominal.

Cet adjectif ne peut pas être ni éliminé ni remplacé, sauf les exemples $n^{\circ} 17$ et 20, où il est postposé. Cela signifie que l'adjectif beau apporte parfois une caractéristique supplémentaire dans le genre du rap. Cet adjectif peut par conséquent posséder non seulement une distinction intérieure mais aussi extérieure de l'objet, comme c'est le cas d'autres adjectifs tels que « ancien, vieux, bon, même, brave, nouveau, pauvre, prochain, sale, seul », qui changent leur place en fonction du sens. Or, cela s'explique non seulement par la volonté de donner une caractéristique inté- 
rieure ou extérieure à l'objet mais aussi par un rythme fréquemment syncopé du rap.

Le cas de l'absence de l'adjectif beau postposé dans la chanson est aussi dû à la quantité moins importante de mots - le rap français possède deux fois plus de mots que la chanson française (41000 vocables pour la chanson et 81000 mots). Une autre remarque serait que l'adjectif beau est néanmoins plus fréquent comme une épithète dans la chanson que dans le rap (56 vs 31 occurrences). Et cela va de soi car le genre de la chanson est plus ancien et lié plus à la poésie que le rap qui est assez récent et plus proche à la vie quotidienne.

\section{Autres cas de l'utilisation de beau}

Dans les deux genres, nous avons voulu trouver des synonymes de beau. Le plus proche synonyme est bon avec 66 occurrences dans les textes de la chanson (18 cas) et du rap (48 cas) français. En plus, dans les deux genres musicaux, l'adjectif bon joue le même rôle d'épithète (38 cas) et d'attribut (11 cas). Il est toujours accordé avec son complément d'objet direct, par ex. :

(21) Le jour où je n'ai pas croisé la bonne fée. (Suprême NTM, J'appuie sur la gâchette) ;

(22) Pas de bon thé sans théière. (Pas De Ci, Fabulous Trobadors).

Dans les exemples n ${ }^{\circ} 1$ et 22, on voit que cet adjectif est aussi antéposé étant épithète et formant le même syntagme nominal. Si nous le retirons, nous perdrons le signifié. Cependant le vocable bon est parfois utilisé comme un adverbe en signifiant « se sentir bien », par ex. :

(23) Pour être en bonne santé (Mignon Mignon, René la Taupe);

ou une interjection, par ex. :

(24) Je ne peux pas faire l'enfant mais bon ce n'est pas... eh revient! (Stromae, Formidable).

On voit donc que la valeur syntaxique de bon est plus large que celle de beau mais son emploi est moins fréquent dans le discours musical français. Cela s'explique probablement par une certaine usure de ce vocable dans la langue française actuelle où il est parfois considéré comme un mot «parasite », qui n'a pas de signification particulière et qui sert à remplir des pauses dans une conversation.

Un autre synonyme de beau est agréable, qui présente une satisfaction et qui est mis deuxième après l'adjectif bon dans le dictionnaire des synonymes consulté (cf. : http://www.crisco.unicaen.fr/des/). Mais nous n'avons trouvé aucun cas avec cet adjectif. L'adjectif joli, qui occupe la troisième position comme un synonyme (ibid.), a été trouvé dans 12 occurrences : 8 cas dans le rap et 4 cas dans la chanson, par ex. :

(25) Je serais devenue jolie. (Jeanne Mas, En rouge et noir); 
(26) Le Papa pingouin poursuit son joli rêve. (Pigloo, Le Papa pingouin).

(27) À de jolies mannequins très convoités. (Doc Gynéco, Nirvana)

Dans les exemples $\mathrm{n}^{\circ} 25$ et 27 , le mot joli concerne des personnes concrètes. Dans l'exemple $\mathrm{n}^{\circ} 26$, l'adjectif joli parle d'un rêve. Il est moins fréquent que l'adjectif beau car plus attaché à un aspect qui vise un plaisir, un agrément et une satisfaction esthétique ou physique. En ce qui concerne l'emplois d'autres synonymes, ce sont des cas uniques et par conséquent peu présents dans le discours musical français.

\section{Conclusions}

Dans cette étude, nous nous sommes penchés sur l'emploi de l'adjectif beau dans le discours musical français moderne. Les données statistiques nous ont permis de voir les cas dans lesquels cet adjectif est utilisé le plus souvent : épithètes et attributs. Cependant, cet adjectif n'a pas de valeur dominante - il apparaît autant dans le rôle d'épithète et d'attribut.

Les données statistiques nous ont autorisé à conclure que l'adjectif beau entrait dans des champs sémantiques très riches dans son usage : parties $d u$ corps, univers artistique, noms propres, gens, environnement, et. La multitude des champs sémantiques démontre son aptitude à s'adapter au rythme et à la mélodie, surtout dans le genre du rap où l'adjectif beau peut être postposé et antéposé.

Dans le langage de la musique, cet adjectif est utilisé pour décrire et souligner le nom qu'ils suivent ou précèdent. L'adjectif beau est nécessaire car il est essentiel pour les textes poétiques même s'il est plus souvent utilisé dans les paroles de la chanson pour décrire un objet ou un être exprimant la discontinuité.

Notre recherche pourrait initier des études contrastives plus larges et détaillées portant sur des paroles des chansons et du rap français en visant des questions de la grammaticalisation et des champs sémantiques des locutions adjectives beau en y incluant d'autres adjectifs, aussi répandus dans la langue française.

\section{Bibliographie}

1. Charaudeau P., Maingueneau D. (2002). Dictionnaire d'analyse du discours. Paris.

2. Ganette G. (1987). Romances sans paroles, musique et littérature. Ed., Revue des Sciences Humaines, no 205.

3. Grevisse M. (2003). Précis de grammaire française. Edition Duculot, Bruxelles.

4. Marquet M. (2013). Politisation de la parole : du rap ludique au rap engagé, variations [http://journals.openedition.org/variations/645] 
5. Nietzsche F. (1879). Humain, trop humain. Euvres Robert Laffont - Bouquins, Paris.

6. Nikolskaia E.K., Goldenberg T.Y. (1974). Grammaire française. Editions école supérieure, Moscou.

7. Pougeoise M. (1996). Dictionnaire didactique de la langue française, Paris.

8. Référovskaia E.K., Vassilieva A.K. (1973). Essai de grammaire française. Moscou-Leningrad.

1 Larousse (https://larousse.fr/dictionnaires/francais)

2 Le Dictionnaire Électronique des Synonymes (http://www.crisco.unicaen.fr/des/ ) 


\title{
THE PLACE OF THE ADJECTIVE "BEAUTIFUL" IN THE FRENCH SONGS AND RAP
}

\author{
Dr. Miroslav Stasilo \\ General Jonas Žemaitis Military Academy of Lithuania \\ Vilnius University
}

\section{Summary}

The study deals with the occurrences of the qualifying adjective beautiful frequently used in the French language. Based on the digital corpus, which includes a database of approximately 122,000 French words, including 41,000 song words and 81,000 rap words, the article presents a variety of uses and differences of this adjective concerning its syntactic functions and the semantic values that they acquire in the French musical discourse. For the analysis of the occurrences, the software Notepad++ was used for a lexicometric treatment of 240 most popular singles sold in France between 1989 and 2019 (4 songs each year). The results of the research demonstrate that the adjective beautiful was more variable than other adjectives not only as a tool for expressing a particular quality of an object but also as an inseparable constituent of its noun phrase. The study also presents a variable place of the adjective, generally placed before the noun, in the French rap.

Keywords: adjective qualifier, song, rap, quantitative analysis, semantic fields, parsing. 


\section{AUTORIAUS LYDRAŠTIS}

Autoriaus vardas, pavardè: Miroslav Stasilo

Mokslo laipsnis ir vardas: filologijos mokslų daktaras

Darbo vieta ir pareigos: Lietuvos karo akademijos Humanitarinių mokslų katedra; Vilniaus universiteto Filologijos fakulteto Taikomosios kalbotyros institutas

Autoriaus mokslinių interesų sritys: užsienio kalbos, sociolingvistika, politikos mokslai

Telefonas ir el. pašto adresas: + 3706 1144811;

francaisprancuzas@yahoo.fr

\section{AUTHOR'S COVER LETTER}

Author's name and surname: Miroslav Stasilo

Academic degree and name: Doctor of Linguistics

Workplace and position: Department of Humanities, General Jonas Žemaitis Military Academy of Lithuania; Faculty of Philology, Applied Linguistics Institute, Vilnius University

Author's research interests: foreign language, sociolinguistics, political science

Telephone and e-mail address: +37061144 811;

francaisprancuzas@yahoo.fr 